\title{
Individu, identité et interculturalité dans Mission terminée de Mongo Beti
}

\author{
Individual, Identity and Interculturality in Mongo Beti's Completed \\ Mission
}

Ibrahim Boumazzou

Université Ibn Tofail, Maroc

Résumé : Cet article se veut une étude analytique de l'identité et de la culture africaine dans Mission terminée de Mongo Beti. Il a pour objectif de faire la lumière sur la spécificité de cette société, la place que l'individu y occupe et les transformations qu'elle a subies avec l'arrivée du colonisateur. II est centré d'abord sur les personnages du roman et leurs attributions afin de savoir comment est constitué, structuré et hiérarchisé le groupe social auquel appartiennent ces entités. II reconstitue ensuite les aspects culturels de la vie de l'homme noir tels qu'ils sont suggérés par le texte.

Mots-clés : colonisateur, identité, culture africaine, Mongo Beti.

\begin{abstract}
This paper is intended to be an analytical study of African identity and culture in Mongo Beti's Completed Mission. It aims to shed light on the specificity of this society, the place that the individual occupies there and the transformations that it suffered with the arrival of the colonizer. It focuses first of all on the characters of the novel and their attributions in order to know how the social group, to which these entities belong, is constituted, structured and hierarchized. It then reconstructs the cultural aspects of black man's life as suggested by the text.
\end{abstract}

Keywords: colonizer, identity, African culture, Mongo Beti.

La société africaine est au cœur de l'œuvre romanesque de Mongo Beti. Dans Mission terminéel, celui-ci décide non seulement de la décrire dans sa réalité quotidienne et globale mais aussi de brosser un tableau scénographique des mœurs, des institutions sociales, des identités et des rapports entre les hommes qui l'animent. La confrontation entre deux visions du monde, deux mondes totalement différents y trouve également une place : Jean-Marie Medza, jeune lycéen ayant toujours vécu en ville et venant d'échover au baccalauréat, découvre avec émerveillement les richesses culturelles de la tradition en même temps qu'il se rend compte de l'inadéquation de l'éducation reçue à l'école coloniale alors que les villageois, bien que critiques, voient en lui le symbole de la réussite et de l'avenir.

\footnotetext{
1 Mongo Beti (1957): Mission Terminée. Paris : Buchet-Chastel. Toutes les citations se rapportent à la présente édition.
} 
Le roman sur lequel porte l'analyse dans cet article met en scène les pérégrinations de Medza à qui est confiée une délicate mission, celle d'aller chercher la femme de son cousin Niam. Celle-ci vient d'abandonner le lit conjugal pour aller se réfugier dans son village maternel après une brouille avec son époux. C'est ce cheminement, cette aventure qui donne lieu à l'initation du protagoniste tout en l'ouvrant à de nouvelles conceptions de la réalité africaine, plus précisément celle du monde du village.

\title{
L'homme noir de Kala ou l'image du bon sauvage²
}

Après avoir été recalé à l'oral de son baccalauréat, le jeune Medza rentre dans son village natal. Mais à peine arrivé, on lui demande de repartir chercher la mariée disparue. Selon les antiques traditions du village, cette mission sera pour lui le passage obligé pour renouer avec sa communauté et assumer ses responsabilités envers elle. Medza ne l'acceptera pas facilement. II ne le fera qu'après une longue négociation avec Niam, soutenu par Bikoloko le patriarche, et avec, comme public, les autres habitants du village. Un de ses oncles le met en cause devant l'auditoire en lui rappelant ce que disent les coutumes et la sagesse qu'il a perdues:

\begin{abstract}
Ce garçon n'a peut-être rien compris à rien ; il n'a certainement rien compris. [...] Pourquoi voulez-vous qu'il comprenne forcément ? Je vous le demande. Songez donc, depuis qu'il ne vient parmi nous que par intermittence, depuis qu'il est à l'école, le miracle, ce serait bien plutôt qu'il soit encore à l'aise dans notre sagesse et dans nos coutumes. Au lieu de vous étonner, expliquez-lui donc (p. 27).
\end{abstract}

Selon son oncle, c'est la formation de Mezda à l'école coloniale qui en est la cause. Celle-ci a accentué la séparation de l'adolescent d'avec sa communauté. Les jeunes y apprenaient telles quelles les leçons dans des livres destinés aux petits enfants du colonisateur. De cette façon, l'enseignement devient une négation de toute civilisation, tentant de rendre français des gens qui ne pourront jamais le devenir. Pourtant, le fait d'avoir assimilé des matières « européennes " non adaptées à la vie des villageois semble avoir particulièrement ému le patriarche. II adresse donc des explications à Medza:

\begin{abstract}
Mais tu es un homme terrible! E† tu parles aussi avec la voix du tonnerre. E† tu ne soupçonnes même pas ta puissance! Ta voix du tonnerre, sais-tu ce que c'est ? Tes diplômes, ton instruction, ta connaissance des choses des Blancs. Sais-tu ce que s'imaginent sérieusement ces bushmen de l'arrièrepays? Qu'il te suffirait d'adresser une lettre écrite en français, de parler en français au chef de la subdivision la plus proche, pour faire mettre en prison qui tu voudrais... Voilà ce que s'imaginent ces péquenots chez lesquels nous t'envoyons (p. 28).
\end{abstract}

Dans son village, le recalé au baccalauréat endosse le costume et la posture d'un conquistador. Tel un Pizarro, ou un carbonaro ${ }^{3}$, il se fraie un chemin dans ce pays

\footnotetext{
2 Figure mythique qui est née justement de la rencontre de la population amérindienne et des aventuriers espagnols, partis à la conquête de l'Amérique au XVle siècle. Voir à ce sujet l'ouvrage de Bernard Mouralis, Montaigne et le mythe du bon sauvage. De l'Antiquité à Rousseau, Paris : Bordas, 1999.

3 Membre d'une société secrète italienne qui, au début du XIXe siècle, combattait pour la liberté nationale.
} 
tant désiré. II contemple donc l'immense panorama ouvert à ses futurs exploits et voit s'accomplir la prédiction du patriarche :

Dès l'abord, il me fut donné de vérifier à quel point le terme péquenot, utilisé le matin par Bikokolo, le patriarche, s'appliquait aux gens de ce pays. Juste à l'entrée du village se déroulait un spectacle saisissant non tant par son décor que par la rude sauvagerie qui en agitait tous les acteurs ( $p$. 37).

Medza marque une position par rapport aux habitants de Kala et les rejette en les taxant de sauvagerie, reproduisant ainsi les poncifs romantiques de l'Africain-bon sauvage qui vit au plus près de la nature. Dés son arrivée, il les décrit en train de pratiquer, "le torse et les jambes nus ", un sport inconnu évoqué à travers des expressions comme : "cette espèce de baobab humain qui lançait la boule... ", ou encore: "la boule lancée par cette force d'orang-outang ", ou " tous les jeunes gens prirent le large à l'approche du rhinocéros vert, sauf Zambo qui, gambadant sur place, comme une antilope géante [...] ॥. Medza prend rapidement conscience d'une autre Afrique, d'une Afrique qu'il ne connaissait pas, plus pure et moins touchée par la civilisation européenne, où les jeunes sont libres, sans tabous sexuels, indisciplinés, gais, se prodiguant une amitié franche et solide.

Cette image de l'Afrique-nature devient une sorte d'identité plurielle propre aux habitants naïfs de Kala. Tout au long de son séjour dans ce village, le narrateur ne cessera de la leur attribuer. Dans le roman, nous le remarquons à travers l'emploi des thématiques stéréotypées de la nudité et de la débauche, de la paresse et de la danse, du paysage naturel et de l'esprit bon vivant, de la naïveté d'un côté, et de la sagesse, de l'autre.

Quant à l'identité de Medza, elle ressemble un petit peu à celle d'un "étranger » ou de "l'inadapté à la société, qui n'est jamais à sa place là où il est, et qui en change perpétuellement ॥, qui débarque de loin. Au milieu de cette vie plus saine et plus proche de la nature, Jean-Marie, à son grand étonnement, apparaît non seulement comme un diplômé, mais aussi comme un " rupin ", un " gars de la ville ", un « blanc », voire un « colon ».

Nous assistons donc à deux identités différentes, celle du protagoniste qui est investi d'une mission par le chef de la tribu et celle des autres personnages. D'ailleurs, le narrateur ne cesse d'insister sur son altérité culturelle, par exemple quand il raconte comment il a pris Edima pour épouse sans en être conscient, et cela en raison de son ignorance des codes de la cérémonie traditionnelle du mariage ; ou quand il raconte comment, par pudeur, il se baignait dans la rivière avec un slip, alors que ses amis de Kala "étaient complètement nus ". Le narrateur se démarque du groupe par la honte qu'il éprouve à l'idée de sa nudité. Derrière son embarras d'adolescent et la situation comique qui en résulte, l'action de se couvrir prend un sens symbolique : elle positionne le narrateur à l'écart des autres qui restent dans le même état d'innocence édénique d'avant la chute.

Medza prend d'autres postures identitaires lorsque par exemple un parterre d'auditeurs lui demande de parler de ce qu'il a appris à l'école des Blancs:

Pour nous, lui dit-on, [qui] n'avons pas été à l'école, le Blanc, c'est toi fils, parce que toi seul peux nous expliquer tout ce que nous ne comprenons pas. Fais-le par amour pour nous, fils. Si tu refusais, nous aurions 
probablement manqué la seule chance que nous n'ayons jamais eve de pouvoir comprendre (p. 86).

Medza se met dans la peau d'un professeur dont la mission civilisatrice consiste à faire sortir le sauvage noir de Kala de son ignorance. C'est ce qui lui permet d'élaborer le discours suivant :

Eł me voilà parti sur les tracteurs, les sovkhozes, les communautés rurales, leur gestion, les résultats magnifiques [...] Mon auditoire haletait. À un moment, je pris une pause pour souffler, tant je m'étais épuisé en parlant : enseigner n'est décidément pas une sinécure (p. 88).

Par ce discours, le narrateur endosse, cette fois, les habits d'un sociologueanthropologue qui étudie les institutions et les mœurs de cette société africaine détachée de la civilisation. C'est, bien entendu, cette posture qui lui confère le droit de faire des observations, par exemple, sur la place de l'individu dans la société, comme c'est le cas dans cette phrase : "Ici, I'individu obéissait au groupe avec une complaisance qui n'eût pas manqué de surprendre Lévy-Bruhl lui-même "); ou sur l'attitude contestataire de la nouvelle génération devant " la chefferie traditionnelle ", souvent appuyée par le régime colonial en place : "opposition anarchique, certes, verbeuse, mais qui n'en portait pas moins de fruits... "..

Les rares connaissances de Jean-Marie deviennent donc des mythes en pays Kala. Sachant les " secrets" des Blancs, il en est le représentant dans ce village de brousse. Lui qui n'est rien aux yeux d'un Européen, se voit mis sur un piédestal chez des gens plus ignorants que lui. Les questions pleuvent, et ses réponses, plus ou moins maladroites, montrent encore plus l'immense écart entre les mentalités africaines et européennes. Pour les Africains, le savoir suprême est celui acquis patiemment par les enseignements ou les traditions des ancêtres. Ils ne voient donc pas pourquoi leurs enfants ne seraient pas aussi bons que les enfants blancs, étant donné que tous les secrets leur sont enseignés à l'école coloniale.

La posture identitaire d'étranger que notre héros a construit tout au long de son récit lui permet de conclure que "les péquenots de Kala " sont des "sortes de caricatures de l'Africain colonisé " qui est " laissé à lui-même dans un monde qui ne lui appartient pas, un monde qu'il n'a pas fait, un monde où il ne comprend rien ॥.

Au terme de son voyage à Kala, Medza ne retrouve donc pas son village natal pour y vivre heureux le reste de ses jours. II n'y réside que très peu de temps avant de rompre définitivement avec sa famille et son épouse. Malgré son niveau d'instruction, son séjour à Kala en a fait un homme détraqué, ne trouvant plus de milieu qui pourrait le comprendre et l'intégrer; il refuse aussi bien la vie traditionnelle (il aurait pu vivre paisiblement à Kala avec Edima son amour) que les avantages que pourrait lui offrir sa réussite scolaire. "Je ne veux plus aller au collège, je ne veux plus me présenter à aucun examen 11 , crie-t-il dans sa crise d'exaspération devant l'attitude répressive de son père. Ce refus catégorique l'éloigne à tout jamais de la tradition et le jette dans une errance sans fin. II en parle ainsi :

Cette imperturbable sérénité devant les vicissitudes éventuelles de la vie, c'est probablement la plus grande perte que nous ayons faite, nous de la ville, en quittant nos villages, nos tribus, nos cadres; car nous ne l'avons plus, cette sagesse : irrités, ambitieux, pleins d'illusions, exaltés, nous sommes les dupes éternelles (p. 216). 


\section{Deux époques, deux cultures}

Dans Mission terminée, il est non seulement question d'identités mais aussi de cultures différentes. Celle des habitants de Kala est dite traditionnelle et originelle parce qu'elle indique les origines. Quant à celle du colonisateur, elle est moderne et on la qualifie souvent de culture de l'autre. L'homme noir de Kala se trouve tiraillé entre les deux et sa psychologie en est tellement affectée qu'on assiste à une perte partielle et/ou totale de son identité. II est complètement désorienté car il ne sait plus vraiment où est sa vraie place, dans la première ou dans la deuxième.

C'est Medza, le personnage central du récit, qui incarne le mieux ce tiraillement. Son déplacement de la ville vers le village montre qu'il souffre d'une perte d'identité. À Kala, il ne comprend que peu de choses à la vie des habitants car il a fait toutes ses études dans les écoles coloniales. II n'était donc en contact avec leurs traditions que rarement, plus précisément pendant les vacances, quand il rentrait chez lui.

Devant les autres, Medza se comporte exactement comme un Blanc. C'est le cas dans l'épisode du bus situé au début du roman où le narrateur rapporte la discussion de l'adolescent avec le chauffeur grec. Chose considérée comme inimaginable par tous les voyageurs : " un Noir ne doit jamais s'engager dans un débat avec un Blanc $॥$, disent-ils.

À cause de l'arrivée des Blancs à Kala, la mentalité des noirs aussi bien que leur culture ont subi un profond changement. La fierté n'est plus alors d'être un simple villageois, mais d'être une personne qui ressemble aux Blancs. II est donc impératif de changer de mode de vie si l'on veut réussir dans la vie et devenir riche. C'est pourquoi les parents poussent leurs enfants " comme on pousse des troupeaux vers un abattoir " vers les établissements coloniaux afin qu'ils puissent bénéficier de tous les atouts offerts par ceux-ci. De cette manière, une partie de l'identité des gens de Kala se voit effacée laissant place à une autre, toute nouvelle. Tel est le prix à payer pour réussir.

C'est ce qui arrive à Medza. Nous pouvons dire que son voyage est une quête de l'identité perdue à cause de l'école. Grâce à ses aventures à Kala, il a pu, pour la première fois, découvrir la joie de vivre en fréquentant des adolescents du même âge que lui. En effet, il a goûté au sexe, à l'alcool et aux fêtes. Aussi a-t-il pu savoir ce que c'est que l'hospitalité des gens de l'arrière pays et la solidarité grâce à ce qu' on appelle " communauté de sang ", sujet sur lequel son oncle Mama s'étendra assez longtemps pour lui signifier que c'est là un point fondamental dans les rapports humains :

À croire qu'à ses yeux, tout se ramenait à la communauté de sang. Ce devait être pour lui la clé de toute science, le fin mot de tout mystère, la base de sa conception de l'univers, sa théorie d'Euclide, ou mieux sa quatrième dimension. D'autres avaient inventé la relativité de l'univers, la géométrie à courbures positives et négatives, lui, avait trouvé ça : la communauté de sang, cosmogonie irremplaçable, irréfutable, inconstatable (p. 114).

Dans la culture africaine, cette relation traditionnelle lie involontairement les individus de la tribu et ceux des autres tribus par le sang, même sans lien de parenté. Grâce à elle, Medza ne manque donc de rien à Kala. II est bien nourri et bien 
hébergé. Il est très estimé et est considéré comme un dieu favorable : on ne jure plus que par son nom dans chaque foyer.

C'est aussi à Kala que Medza découvre vraiment le sens de la vie traditionnelle. Pour lui, elle est tout à fait différente de ce qu'il a vécu en ville. Pendant tout son séjour, il a pu observer à quoi se résume le quotidien des villageois. Pour manger à leur faim, ils sont obligés de travailler dans les champs à la machette du matin jusqu'au soir. C'est une vie dure et monotone, mais elle fascine Medza car, comparé à ce qu'il a enduré en ville, la vie à la campagne le rend heureux. Elle est le symbole de la pureté et le dernier paradis sur terre. C'est au contact de cette terre profonde que le héros découvre diverses richesses et se rend compte des limites du mode de vie et de pensée des Blancs. Une fois à Kala, Medza prend conscience des insuffisances du système scolaire. La découverte du monde traditionnel est pour lui une sorte de renaissance, une seconde école de vie plutôt complémentaire et nécessaire à l'école coloniale, comme il l'avoue dans le passage suivant:

Plus j'y pense et plus je me dis que c'est certainement moi qui devrais savoir gré pour ce voyage qui m'a permis entre autres choses, de découvrir, au contact des péquenots de Kala [...] le drame d'un homme sans direction intellectuelle, d'un homme marchant à l'aveuglette, la nuit [...] (p. 219).

Le village africain est encore éloigné de la modernité et de ses problèmes. Kala ne va pas échapper à cette modernisation, un commencement de déformation de la culture originelle se perçoit déjà. Ainsi, le séjour de Medza aura contribué à l'évolution de sa conscience, mais cette évolution reste sans issue pratique. Comme il l'avait lui-même prévu, il aura connu des " aventures sans précédent dans (sa) vie ", décisives du reste. Le jeune Medza n'aura même pas le courage d'utiliser ce qu'il sait des deux réalités pour essayer de construire quelque chose de neuf, de solide, de positif. II fuit, et il semble qu'il fuira toute sa vie, étant donné qu'il a découvert «le drame dont souffre son peuple», drame dont il souffre lui-même.

\section{L'ethos du père africain dans la culture ancestrale}

Le personnel romanesque betien comprend une multitude de personnages de chefs qui sont tous présentés, sans exception aucune, comme des freins à la modernité, des partisans de l'immobilisme refusant toute évolution au nom d'une prétendue tradition. Tel est plus généralement le cas de la figure du père. Ce dernier jouit d'un respect absolu et peut faire ce qu'il veut de tous les autres membres de la communauté, plus précisément des femmes et des enfants. Les maltraiter fait partie intégrante de la culture:

[...] chez nous, explique le narrateur, on se tançait sans arrêt : le père tançait tout le monde, la mère tançait les enfants, [...] Quant aux enfants, les garçons tançaient les filles, et la fille aînée tançait sa cadette [...] (p. 33).

Tout au long de Mission terminée, l'homme ne fait que chasser, pêcher, donner des ordres et battre les femmes. Aux autres de les respecter. Tel est l'exemple de Bikololo, l'homme sage du village qui exerce un pouvoir absolu sur tous les individus de la tribu. II assure plusieurs fonctions. II est, à la fois, dirigeant, conseiller et guérisseur. Ses décisions ne doivent jamais être contestées par qui que ce soit. L'oncle Mama, de son côté, est un homme ferme. Dans son foyer, il parle peu et ne rit jamais. II impose ce qu'il veut sans que les autres membres de sa famille aient le temps de réagir. Medza, le personnage central du récit, accuse les hommes du 
roman, notamment son père, d'être autoritaires et despotiques. Depuis son enfance, il n'a fait que suivre les désirs d'un père que tout le monde craint.

À Kala, le père est " un dieu suprême, un dieu terrible ». Au moment du repas, il mange seul et ne se mêle pas aux femmes, considérées comme des êtres inférieurs. Lors de la baignade, il est inadmissible pour les hommes que les femmes aient une place à leur côté. La supériorité de l'homme est donc incontournable. À cause du "système économique, juridique et coutumier ", les hommes sont privilégiés. Ils tiennent les autres classes de la société dans une totale sujétion. Même les jeunes du roman ne peuvent pas les rivaliser. Ils se plient à leurs exigences.

Pour se libérer du joug paternel, Mezda se révolte. Mais son action semble naître d'un élan individuel face à un déni de justice qui le touche personnellement. Elle est donc d'emblée vouée à l'échec. La société africaine restera toujours une société patriarcale où «il y a des gens qui ne perdent jamais » et où les plus forts dominent les plus faibles et les moins âgés. C'est presque un constat que dresse le narrateur au début du roman :

Peu à peu se formait en moi la conviction que ces menus événements joveraient dans mon existence un rôle hors de proportion avec leur signification d'alors, qu'ils marqueraient comme un tournant dans ma vie (p. 16).

\section{Acculturation de l'éducation et du mariage}

L'éducation et le mariage sont les deux institutions sociales qui ont été profondément transformées dans la vie des habitants de Kala après l'intrusion de la culture européenne. En effet, les parents sont devenus de plus en plus attachés à la culture du colonisateur. Ils ont délaissé l'éducation traditionnelle mais en revanche, ils sont prêts à tout pour pouvoir envoyer leurs enfants à l'école française. Ils croient que ceux-ci réussiraient dans la vie s'ils arrivaient à intégrer cet établissement.

Les connaissances délivrées par les vieux de la brousse autant que leurs conseils ne constituent plus une priorité ni pour les parents ni pour leurs enfants. Ces derniers s'en désintéressent complètement. Dès qu'ils rencontrent Medza, le nouvel enseignant de Kala, ils se ruent vers lui pour apprendre à lire et à écrire. Ils ont tellement soif de nouvelles connaissances qu'ils viennent chez lui avec le matériel nécessaire comme les livres et les ardoises.

Medza prend la place des vieux et joue leur rôle. II occupe une place privilégiée car il est un intellectuel. Son statut de citadin et l'auréole de gloire que lui confèrent ses années d'étude à l'école des Blancs en font, pour les "péquenots de Kala », un individu important. II se fait toujours inviter par différents pères de famille pour animer des conférences. Chaque fois, l'auditoire gagne en nombre et en variété puisque tout le village est mobilisé pour l'occasion. On s'intéresse beaucoup aux informations nouvelles apportées et développées par lui. Les veillées répétées auxquelles est soumis Medza par les habitants de Kala témoignent de la volonté de comprendre et de connaître. Chaque membre épie l'occasion et cherche à saisir et à déceler les fondements des explications données par le protagoniste. Les questions qui lui sont posées le prennent au dépourvu. Medza ne manque pas de faire état de sa gêne:

Un homme que je n'ai pas identifié me pose une question fort embarrassante, tentant de me contraindre à m'expliquer sur un point 
auquel mon esprit ne s'était jamais arrêté et dont l'idée m'eût terrorisé, je veux dire mon avenir ainsi que celui de tous ceux de ma génération (p. 88).

Son embarras grandit au fur et à mesure que les débats s'approfondissent :

Décidément, voici la soirée des surprises. Moi qui avais préparé de jolies définitions, m'imaginant que cette séance ressemblerait à celle de la veille, voilà qu'encore une fois, la vie me trahissait : je ne me trouvais que devant des questions qui me prenaient de court (p. 110).

C'est le cas également de la cérémonie de mariage du chef. Ce dernier reçoit en personne l'oncle Mama, Zambo et Medza. Ils sont considérés comme des invités d'honneur. Le chef est allé jusqu'à leur donner les meilleures places et veille à ce qu'ils ne manquent de rien. En témoigne l'extrait suivant:
À notre entrée, le chef se leva avec empressement et vint à notre rencontre, comme si nous avions représenté auprès de sa majesté une grande puissance digne de toutes les préséances. [...] les gens s'étaient tus en nous voyant arriver. Le chef me promena à travers la salle et me fit toucher la main de tous les assistants [...] (p. 183).

Les questions auxquelles Medza est invité à répondre et la place qu'on lui donne au village montrent qu'on accorde surtout de l'importance à l'avenir non seulement du monde européanisé mais aussi des campagnes. Elles portent sur les rapports scolaires entre les enfants blancs et noirs, sur les matières enseignées aux enfants à l'école, sur les connaissances qu'ils ont du monde extérieur, sur l'avenir des enfants scolarisés et sur leurs rapports futurs avec les Blancs et avec la chefferie traditionnelle.

Pour ce qui est de l'institution du mariage, elle aussi a subi une métamorphose complète. Le narrateur montre qu'elle n'est plus la même depuis l'arrivée de la culture occidentale en Afrique. La polygamie qui y est de tradition en a été affectée. On ne peut plus observer des hommes unis avec plusieurs femmes comme c'était le cas du chef de canton qui " possédait les six plus belles femmes de la région et s'apprêtait à en acquérir d'autres $\|$, et du chef de village de Kala qui " venait d'épouser une femme - sa septième [...] ». On ne peut non plus trouver des femmes qui vivent sous le même toit en parfaite harmonie, sans se plaindre de leur situation.

L'arrivée des Blancs a tout changé. C'est le cas également pour la femme. Celleci commence à revendiquer ses droits. Elle « [...] pouvait [même] quitter son mari - à la condition toutefois de lui rembourser sa dot. "C'est ce qui s'est passé pour l'épouse Niam qui a quitté son mari pour un autre homme. Grâce aux nouvelles lois, son amant n'a qu'à rembourser la dot. Eł s'il n'a pas les moyens de payer en espèces, la somme est convertie en béliers ou en brebis.

Tout le règlement traditionnel du mariage a donc été modifié, rendant " les femmes de Kala désobéissantes, mauvaises épouses 1 . Le colonisateur l'a adapté au nouveau mode de vie : il l'a modernisé sans prendre en compte ni la personnalité ni les origines de la tradition africaine. Sur le plan socioculturel, ce métissage, non pas voulu et conscient, mais conçu et imposé par le colonisateur a eu pour conséquence d'arracher Kala à ses racines, d'en faire un monde à la recherche de son moi. 
Force est de constater que l'auteur de Mission terminée fait plus que suggérer, ne serait-ce qu'implicitement, l'une des situations inhérentes au contexte colonial : les problèmes du contact entre les deux civilisations, africaine et européenne, et le phénomène de l'acculturation par lequel les Blancs s'efforcent d'assimiler les Noirs de Kala, de les déshumaniser en y implantant de gré ou de force leurs propres valeurs, systèmes et culture.

Si la critique de la colonisation est moins directe dans ce roman que dans les autres "romans coloniaux " de Mongo Beti, la portée du sujet est tout aussi conséquente : c'est l'avenir de tout un continent désafricanisé, ne sachant plus à qui il appartient, qui est ici mis en question. Au lecteur de juger et d'établir le moyen terme entre la pensée bétienne et la réalité historique, réalité qui semble chez notre auteur fort significative.

\section{Bibliographie}

AA. VV. (1957) : L'homme de culture noir et son peuple. Paris : Présence africaine, $\mathrm{n}^{\circ} 16$ (octobre-novembre).

ALEXANDRE, P. (1990): L'Afrique, zones culturelles et grandes traditions orales. Le Grand Atlas des Littératures. Encyclopedia Universalis.

BETI, M. (1957) : Mission terminée. Paris : Buchet-Chastel.

BOKIBA, A.-P. (1999): Écriture et identité dans la littérature africaine. Paris : L'Harmattan.

CHEVRIER, J. (1974) : Littérature nègre. Paris : Armand Colin.

DAVIDSON, B. (1962) : L'Afrique avant les blancs. Paris : PUF.

GUILBERT, L. (1975) : La créativité lexicale. Paris : Larousse.

LATOUCHE, S. (1978) : L'autre Afrique. Paris : Albin Michel.

LONDRES, A. (2000) : Terre d'ébène. Paris : Serpent à plumes.

MANNONI, O. (1950) : Psychologie de la colonisation. Paris : Sevil.

MEMMI, A. (1973) : L'homme dominé. Paris : Payot.

MICHAUD, G. (1978) : Négritude : traditions et développement. Paris : PUF.

MOURALIS, B. (1981) : L'œuvre de Mongo Beti. Paris : Les classiques africains. 